\title{
Hypofractionated Radiotherapy With Simultaneous-integrated Boost After Breast-conserving Surgery Compared to Standard Boost-applications Using Helical Tomotherapy With TomoEdge
}

\author{
FELIX ZWICKER ${ }^{1,2,3}$, SEBASTIAN HOEFEL ${ }^{2,4}$, CORINNA KIRCHNER ${ }^{2}$, \\ PETER E. HUBER ${ }^{1,3}$, JUERGEN DEBUS ${ }^{1,3}$ and MICHAEL SCHEMPP ${ }^{2}$ \\ ${ }^{1}$ Clinical Cooperation Unit Molecular Radiation Oncology, \\ German Cancer Research Center (DKFZ), Heidelberg, Germany; \\ ${ }^{2}$ Clinic and Practice of Radiation Oncology/Practice of Radiology Konstanz, Konstanz, Germany; \\ ${ }^{3}$ Department of Radiation Oncology, University of Heidelberg, Heidelberg, Germany; \\ ${ }^{4}$ Department of Chemistry and Konstanz Research School Chemical Biology, \\ University of Konstanz, Konstanz, Germany
}

\begin{abstract}
Background/Aim: This comparative plan study examines a range of boost-radiation methods in adjuvant radiotherapy of breast cancer using helical intensity-modulated radiotherapy with TomoEdge-technique. Impact of hypofractionated radiation with simultaneous-integrated boost (SIB) and influence of differing assumed $\alpha / \beta$-values were examined. Patients and Methods: For 10 patients with left-sided breast cancer each four helical IMRT-plans with TomoEdge-technique were created: hypofractionated + SIB (H-SIB) $(42.4 / 54.4 \mathrm{~Gy}, 16$ fractions), normofractionated $+S I B(N-S I B)(50.4 / 64.4 \mathrm{~Gy}, 28$ fractions), hypofractionated+sequential-boost ( $H$-SB) $(42.4$ Gy/16 fractions $+16 \mathrm{~Gy} / 8$ fractions), normofractionated + sequential-boost (N-SB) (50.4 Gy/ 28 fractions $+16 \mathrm{~Gy} / 8$ fractions). Equivalent doses $\left(E Q D_{2}\right)$ to organs-at-risk $(O A R)$ and irradiated mammary-gland were analysed for different assumed $\alpha / \beta$-values. Results: The mean $E Q D_{2}$ to $O A R$ was significantly lower using hypofractionated radiation-techniques. $\mathrm{H}$-SIB and $\mathrm{H}$ $S B$ were not significantly different. $H$-SIB and $N$-SIB conformed significantly better to the breast planning-target volume (PTV) and boost-volume $(B V)$ than $H-S B$ and $N-S B$. Regarding $B V$, mean $E Q D_{2}$ was significantly higher for all $\alpha / \beta$-values investigated when using $H$-SIB and N-SIB. Regarding PTV, there were no clinically relevant differences. Conclusion: Relating to dosimetry, $H$-SIB is effective compared to standard-boost-techniques.
\end{abstract}

This article is freely accessible online.

Correspondence to: Felix Zwicker, MD, German Cancer Research Centre (DKFZ) E055, Im Neuenheimer Feld 280, 69120 Heidelberg, Germany. Tel: +49 (0)6221422616, e-mail: f.zwicker@dkfz.de

Key Words: Hypofractionated radiotherapy, simultaneous integrated boost, SIB, breast cancer, helical tomotherapy, TomoEdge, IMRT.
Globally, breast cancer is the most common form of cancer in women (1). Surgery and adjuvant radiation therapy are the central pillars of curative therapy when treating localised breast cancer. Screening programs enable tumours to be discovered earlier, meaning that breast-conserving surgery alongside adjuvant radiotherapy achieves high cure rates. Additional radiotherapy significantly increases local control and prolongs overall survival $(2,3)$. As part of adjuvant radiation therapy, a radiation boost to the tumour bed results in significantly improved local control rates in the case of risk factor constellations, such as T2 tumours, positive lymph nodes, premenopausal patients, poorly differentiated G3 tumours, human-epidermal-growth-factor-receptor2 (HER2/ neu) positivity, and narrow resection margins $(4,5)$. The radiation boost can be applied in several ways: teletherapy using photons or electrons, intra-operative radiotherapy, or interstitial brachytherapy (6-9). Thus far, the radiation boost is generally delivered sequentially, i.e., once whole breast radiation therapy is complete. Simultaneous integrated boost (SIB) radiation therapy is a recent approach to delivering radiation boosts (6). SIB can be administered using different percutaneous photon therapy radiation techniques, including 3D conformal radiation therapy (3D-CRT) and intensitymodulated radiation therapy (IMRT). Step-and-shoot IMRT and rotational IMRT, also known as volumetric arc therapy (VMAT), are commonly reported in the literature $(6,10,11)$. The advantages of SIB in comparison to sequential boost (SB) include the reduced total treatment period and the steep dose drop-off between the edge of the planning target volume (PTV) and the remaining breast tissue, which is treated with a lower dose. Thus far, disadvantages include the relatively poor dose homogeneity when using 3D-CRT or IMRT (12). There have also been concerns about the potentially inferior cosmetic results in the long term since SIB supplies higher 
single doses to the PTV for the boost relative to the PTV for the whole breast (13).

Apart from the issue of radiation boosts, new discoveries relating to fractionation, total dose, and treatment length will bring about significant changes to the real-world practice of whole-breast irradiation. In normofractionated breast irradiation, the conventional practice is to administer 25 or 28 daily fractions of $2 \mathrm{~Gy}$ or $1.8 \mathrm{~Gy}$, resulting in a total treatment dose of 50 Gy or 50.4 Gy (12). Large prospective phase III studies have shown that moderate hypofractionation using fractions of 2.5-2.65 Gy and a total dose (TD) of 4042.4 Gy produces equivalent local control and overall survival results to those with normofractionation. Furthermore, hypofractionation significantly reduced treatment time (14-16 fractions) and showed a tendency towards improved cosmetic results. These studies used 3D conformal radiation techniques $(14,15)$.

However, these studies did not apply a radiation boost to the tumour bed in addition to hypofractionated whole breast radiation. Real-world clinical application is based on current indications for a radiation boost following hypofractionated whole breast radiation: sequential normofractionated boost (SB) at 2 Gy per fraction up to a TD of 10-16 Gy. This can be applied using a 3D conformal or IMRT technique (12).

Similar to using SIB alongside normofractionated whole breast radiation, there is discussion around the option to use simultaneous integrated boost alongside hypofractionated whole breast radiation with further increased single doses in the boost PTV (H-SIB). The additional radiation boost in this case would not increase the total treatment period. Concerns about increased fraction doses are related to the potential increase in the risk of late-onset side effects. New data on hypofractionated radiotherapy for whole breast radiation without boost radiation have shown no increase in side effect rates (16-19).

The linear quadratic model can be used to compare radiation exposure in each treatment scheme (20). This shows the difference in doses per fraction and their different biological effects. Doses can be expressed as the equivalent dose in 2 Gy fractions $\left(\mathrm{EQD}_{2}\right)$. A tissue-specific $\alpha / \beta$ factor was used to calculate the equivalent dose. The $\alpha / \beta$ factor itself is determined using radiobiological testing and clinical experience. The precise $\alpha / \beta$ value for breast cancer tissue has not yet been determined; different values have been reported in the literature ranging from 2 to 5 (20). This range of values should be considered in studies of hypofractionated breast radiation, including H-SIB. In this context, there is also interest in radiation techniques that facilitate steep dose gradients between the boost PTV and whole breast PTV or between the whole breast PTV and surrounding normal tissue, where excessively high maximum doses should be avoided to minimise the probability of radiation-induced side effects (21-23).
A relatively new development in percutaneous photon radiation therapy is helical intensity modulated radiotherapy (IMRT), called tomotherapy. In most cases, this technique achieves the steepest dose gradient (conformity index close to 1), lowest values of dose maxima, and best dose homogeneity within the target volume compared to other IMRT and rotational techniques $(24,25)$.

The technical details of helical tomotherapy have been described previously (22). Briefly, a tomotherapy unit is a hybrid comprising a 6-MV linear accelerator and a helical CT scanner. Treatment is applied using a rotating fan beam; as the patient moves through the gantry bore, the treatment beam forms a helix $(26,27)$. The beam is modulated by a very fast-moving, pneumatically driven, binary multileaf collimator (MLC). In an inverse treatment-planning process, the MLC conformation is optimised to obtain highly conformal radiation doses at the target (28). The TomoEdge technique was recently introduced in tomotherapy as a technological development that minimises the dose penumbra at cranial and caudal field borders by modulating the primary collimators. In many cases, this tool shortens treatment duration by factor two with no compromise on plan quality (29).

This comparative plan study examined a range of methods for applying an additional boost in percutaneous breast radiotherapy using helical IMRT (tomotherapy) with TomoEdge. We compared the radiation does exposure to the mammary gland tissue and surrounding organs at risk (OAR). We focused on the effect of H-SIB and on uncertainty regarding $\alpha / \beta$ values for breast cancer tissue observed in the literature.

\section{Patients and Methods}

Patients. Ten patients with left-sided, nodal negative breast cancer were enrolled in this comparative plan study. All patients underwent breast-conserving surgery and were indicated for adjuvant radiotherapy (RT). The study was conducted according to the World Medical Association Declaration of Helsinki and the ICMJE Recommendations for the Protection of Research Participants and approved by the ethics committee of the Landesärztekammer Baden-Württemberg, Stuttgart, Germany (AZ: F-2015-063).

Imaging and regions of interest (ROIs). Patients were imaged using a CT scanner with a slice thickness of $5 \mathrm{~mm}$. A wing step (breast tilting board) and leg fixation were used for optimal positioning. The scans were performed with the patients breathing freely with lifted arms. The left and right lungs, right breast, whole heart, and left ventricle were all delineated on CT images as OAR.

Target volumes were defined according to the institutional standards; the PTV of the treated breast included the whole mammary gland and chest wall behind it with a safe lateral, cranial, and caudal margin of $2 \mathrm{~cm}$ and a safe medial margin of $1 \mathrm{~cm}$. The planning target volume of the boost volume (BV) included a tumour bed defined by surgical clips, architectural distortion, or seroma with a safe margin of $8 \mathrm{~mm}$ in all directions. The BV was always 
inside the PTV. A 1-cm-thick auxiliary structure (shell) was created around the BV to evaluate the dose gradients.

Treatment schemes. Five different treatment plans were generated for each patient. A normal-fractionated plan $(\mathrm{N})$ and a hypofractionated plan $(\mathrm{H})$ for PTV irradiation, a normal fractionated plan for SB, as well as a normal fractionated and hypofractionated plan for SIB. These five plans resulted in four different treatment schemes: a) H-SIB: cumulative dose of 42.4 Gy (PTV) or $54.4 \mathrm{~Gy}$ (BV) in 16 fractions of 2.65 Gy or 3.4 Gy single doses, respectively. b) normofractionated radiotherapy with simultaneous integrated boost application (N-SIB): cumulative dose of 50.4 Gy (PTV) or $64.4 \mathrm{~Gy}(\mathrm{BV})$ in 28 fractions of $1.8 \mathrm{~Gy}$ or $2.3 \mathrm{~Gy}$ single doses, respectively.

c) hypofractionated radiotherapy with sequential boost application (H-SB): cumulative dose of 42.4 Gy (PTV and BV) in 16 fractions of 2.65-Gy single doses plus $16 \mathrm{~Gy}$ (BV) in eight fractions of $2 \mathrm{~Gy}$ single doses.

d) normofractionated radiotherapy with sequential boost application (N-SB): cumulative dose of 50.4 Gy (PTV and BV) in 28 fractions of 1.8 Gy single doses plus 16 Gy (BV) in eight fractions of 2 Gy single doses.

Plan creation. All plans were generated for a tomotherapy planning system using TomoEdge (Version 5.1.1.6). This is a helical IMRT system with a 6-MV photon beam and an integrated planning software for inverse planning. A beam field width of $2.5 \mathrm{~cm}$ was applied for all plans, and the calculations were performed using a fine dose grid. The pitch was set to 0.282 (30) and a modulation factor between 2.4 and 3 was applied. The optimisation of each plan aimed to reduce the dose in all OAR (especially the left lung and heart), while covering the PTV and BV with at least $95 \%$ of the prescribed dose. All plans were created by the same person with high expertise to avoid inter-individual factors. The created plans were checked by a second expert according to the four-eye principle.

Plan evaluation. To evaluate the SB plans, tomotherapy planning software was used to calculate the cumulative dose-volume histogram (DVH) for each of the N-SB and H-SB schemes in each patient. All seven plans (H, N, SB, N-SB, H-SB, N-SIB, H-SIB) were exported to R-Studio (R-based analysis software) for further analysis and statistics.

The mean DVH was calculated across all patients for each treatment scheme. The conformity index (CI) was determined for the BV and PTV, including the BV (PTVIB). Where the term 'PTV' is used in further description of the methods and results, it excludes the BV. The CI was calculated as $\mathrm{CI}=\mathrm{V}_{95}(\mathrm{~T})^{2} /\left(\mathrm{V}(\mathrm{T}) \cdot \mathrm{V}_{95}(\right.$ tot $\left.)\right)(31)$, where $\mathrm{V}_{95}$ represents the $95 \%$ isodose of the prescribed dose in the target $[\mathrm{V}(\mathrm{T})=\mathrm{BV}$ or $\mathrm{V}(\mathrm{T})=\mathrm{PTVIB}]$ and the isodose in the total volume $\mathrm{V}$ (tot). The $\mathrm{CI}$ can reach a maximum of one, which means perfect conformity, when $\mathrm{V} 95(\mathrm{~T})$ is equivalent to $\mathrm{V}(\mathrm{T})$. The homogeneity of the PTV and BV is described using the homogeneity index $\mathrm{HI}=\left(\mathrm{D}_{2}-\mathrm{D}_{98}\right) / \mathrm{D}_{50}$, where $\mathrm{D}_{2}, \mathrm{D}_{50}$, and $\mathrm{D}_{98}$ represent the doses covering $2 \%, 50 \%$, and $98 \%$ of the region under consideration, respectively. The optimum $\mathrm{HI}$ is zero, with higher values representing poorer homogeneity.

The linear quadratic model was used to compare the radiation exposure of each treatment scheme. This shows the difference in dose per fraction and their different biological effects. Doses are expressed as equivalent doses in 2 Gy fractions $\left(\mathrm{EQD}_{2}\right)$. The calculation used is as follows: $\mathrm{EQD}_{2}=\mathrm{d} \bullet \mathrm{n} \bullet\left\{(\mathrm{d}+[\alpha / \beta]) /\left(\mathrm{d}_{2}+[\alpha / \beta]\right)\right\}$, where $n$ is the number of fractions, $d$ is the dose per fraction, $d_{2}$ is the reference dose per fraction of $2 \mathrm{~Gy}$, and $\alpha / \beta$ is the radiation response of different tissue types (32). Throughout our analysis, $\alpha / \beta=3$ Gy was used for all OARs, while for the PTV, BV, and shell, different values were used ranging from $2.2 \mathrm{~Gy}$ to $4.6 \mathrm{~Gy}$ in $0.8-\mathrm{Gy}$ increments to reflect the spread of $\alpha / \beta$ values in breast cancer as reported in the literature (20).

The mean dose values for the SB schemes were calculated separately for the main and SB plan, and then summed up afterwards. This meant that the $\mathrm{EQD}_{2}$ could be calculated properly considering the differences in radiation exposure per fraction between the main and SB plans. Boxplots were created to compare different treatment schemes. Averaged absolute values were presented with standard deviation (+/-). Two different schemes were compared directly using paired $t$-tests. All $t$-test results presented are calculated assuming a null hypothesis and a confidence interval of 0.95 . We assumed a statistical significance for results with $p \leq 0.05$.

\section{Results}

Patients. The mean absolute planning target volume (PTV) in the left breast across enrolled patients was $1068 \mathrm{~cm}^{3}$, ranging 427-2332 $\mathrm{cm}^{3}$. The mean absolute BV was $154 \mathrm{~cm}^{3}$ (range $=41-458 \mathrm{~cm}^{3}$ ). An example of the dose distribution for all four treatment schemes is shown in Figure 1.

$O A R$. Figure 2 shows a statistical comparison of the mean dose in terms of $\mathrm{EQD}_{2}$ for all OARs and each treatment scheme. The radiation exposure was similar, and only small differences were observed. The minimal mean dose values for the left lung for each treatment scheme were associated with one patient, which seems to be because she had the smallest PTV and BV of all subjects. The high dose outliers, i.e., the maximum values for H-SIB and H-SB for the heart and left ventricle also belong to one patient. This patient is notable because of the relatively small distance between the BV and the heart.

Table I shows the mean value in terms of $\mathrm{EQD}_{2}$ for each OAR across all patients and results of the t-test comparison of each treatment scheme with H-SIB plans. The lowest values were achieved using hypofractionated treatments $[e . g$. H-SIB left lung: 7.64 \pm 0.97 Gy (standard deviation); heart: $3.25 \pm 0.62 \mathrm{~Gy}$. While both normofractionated schemes applied a significantly higher mean dose to all OAR, no significant differences were found between the H-SIB and $\mathrm{H}-\mathrm{SB}$ plans, except with regard to the left ventricle (2.74 \pm 0.46 Gy vs. 3.02 \pm 0.74 Gy, respectively, $p=0.034)$.

$D V H, C I$, and $H I$. The raw mean DVH (no $\mathrm{EQD}_{2}$ used) for the PTV including the BV (PTVIB) across all patients and for each treatment scheme are illustrated in Figure 3. Independent of the differences in the prescribed dose, a difference in the shape was observed between the SIB and SB plans. The less pronounced edge between the prescribed PTV and BV dose indicates a worse dose gradient for SB schemes. 


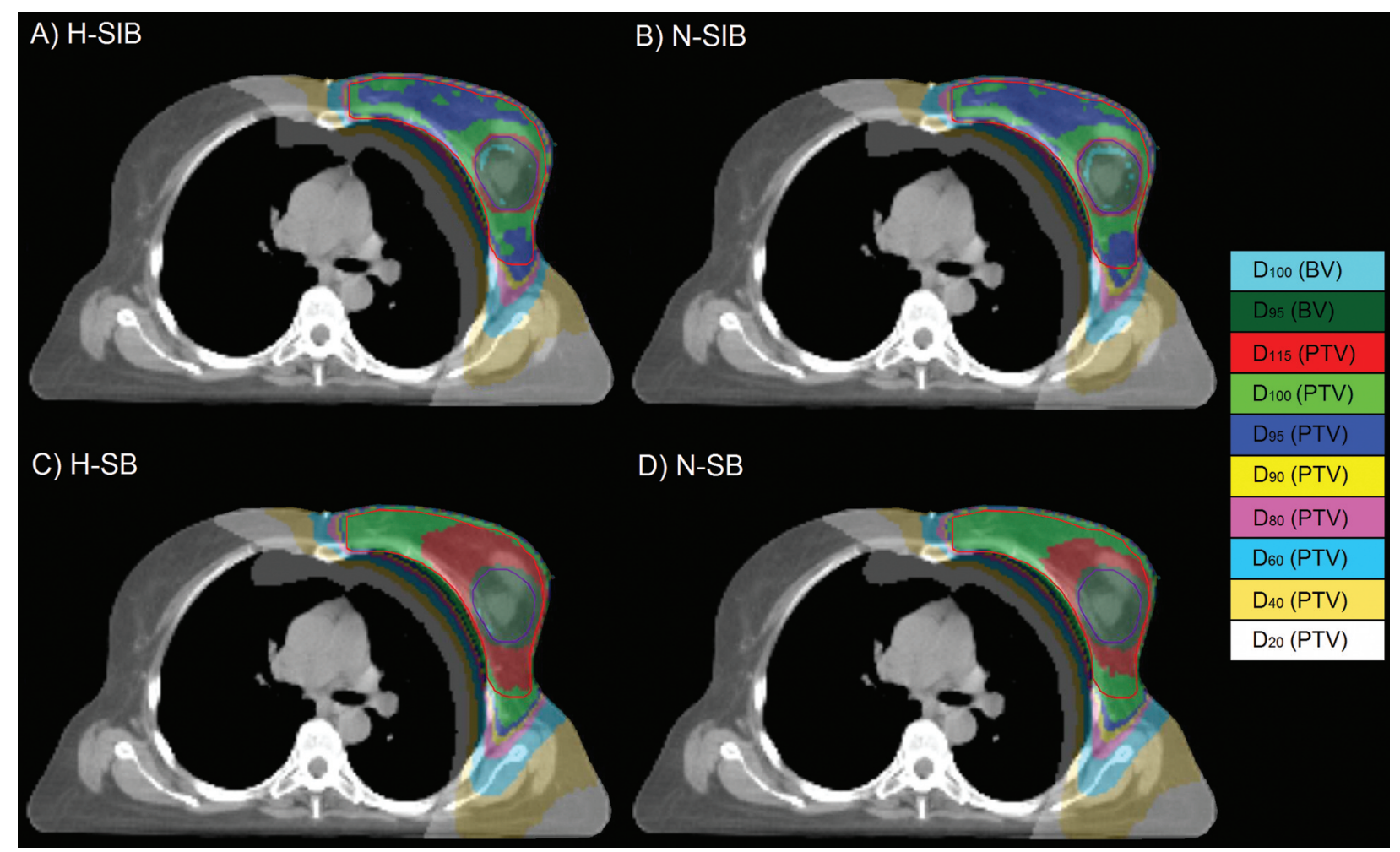

Figure 1. An example of the dose distribution of four different boost application schemes in adjuvant radiotherapy of a female patient with leftsided breast cancer after breast-conserving surgery using helical tomotherapy with TomoEdge technique is shown. In the computer-tomography slice, the planning target volume (PTV) of the whole left breast is marked with red outlines and the boost volume (BV)/ tumour bed is marked with violet outlines. A) H-SIB: hypofractionated radiotherapy with simultaneous integrated boost (SIB) (42.4/54.4 Gy in 16 fractions). B) N-SIB: normofractionated radiotherapy with SIB (50.4/64.4 Gy in 28 fractions). C) H-SB: hypofractionated radiotherapy with sequential-boost (SB) (42.4 Gy in 16 fractions +16 Gy in 8 fractions). D) N-SB: normofractionated radiotherapy with SB (50.4 Gy in 28 fractions +16 Gy in 8 fractions). The relative isodoses of the described $P T V$ and $B V$ doses are shown.

Table I. Averaged mean dose $\left(E Q D_{2}\right.$, calculated with $\left.\alpha / \beta=3 \mathrm{~Gy}\right)$ of organs at risk with standard deviation of all patients and all examined treatment schemes and the p-value of the paired t-test comparison with the H-SIB scheme.

\begin{tabular}{|c|c|c|c|c|c|c|c|c|c|c|}
\hline & \multicolumn{2}{|c|}{ L. lung } & \multicolumn{2}{|c|}{ R. lung } & \multicolumn{2}{|c|}{ Heart } & \multicolumn{2}{|c|}{ L. ventricle } & \multicolumn{2}{|c|}{$\mathrm{R}$. breast } \\
\hline & $\begin{array}{c}\text { Mean dose } \\
\text { (Gy) }\end{array}$ & $p$-Value & $\begin{array}{c}\text { Mean dose } \\
\text { (Gy) }\end{array}$ & $p$-Value & $\begin{array}{l}\text { Mean dose } \\
\text { (Gy) }\end{array}$ & $p$-Value & $\begin{array}{l}\text { Mean dose } \\
\text { (Gy) }\end{array}$ & $p$-Value & $\begin{array}{c}\text { Mean dose } \\
\text { (Gy) }\end{array}$ & $p$-Value \\
\hline H-SIB & $7.64 \pm 0.97$ & --- & $2.34 \pm 0.35$ & --- & $3.25 \pm 0.62$ & --- & $2.74 \pm 0.46$ & --- & $2.91 \pm 0.57$ & --- \\
\hline N-SIB & $8.22 \pm 0.79$ & 0.001 & $2.74 \pm 0.42$ & $<0.001$ & $3.74 \pm 0.93$ & 0.002 & $3.20 \pm 0.65$ & $<0.001$ & $3.49 \pm 0.56$ & $<0.001$ \\
\hline $\mathrm{H}-\mathrm{SB}$ & $7.50 \pm 0.89$ & 0.363 & $2.25 \pm 0.42$ & 0.165 & $3.53 \pm 1.14$ & 0.189 & $3.02 \pm 0.74$ & 0.034 & $2.82 \pm 0.46$ & 0.417 \\
\hline $\mathrm{N}-\mathrm{SB}$ & $8.08 \pm 0.91$ & 0.014 & $2.59 \pm 0.44$ & 0.002 & $3.70 \pm 0.94$ & 0.008 & $3.11 \pm 0.70$ & 0.003 & $3.34 \pm 0.62$ & 0.001 \\
\hline
\end{tabular}

H-SIB: Hypofractionated-simultaneous-integrated boost; N-SIB: normofractionated-simultaneous-integrated boost; H-SB: hypofractionated radiotherapy with sequential boost; N-SB: normofractionated radiotherapy with sequential boost.

The conformity of dose distribution in the PTV and BV was also significantly higher for SIB treatment schemes than for SB schemes, as shown in Figure 4. In particular, the SIB techniques had very high CIs in the BV. The N-SIB plans offered the best conformity (averaged $\mathrm{CI}=0.947 \pm 0.043$ ), but the $\mathrm{CI}$ from the $\mathrm{H}-$ SIB plans was not significantly different $(0.93 \pm 0.032)$ (Table II).

The analysis of homogeneity, shown in Figure 5 and Table II, yielded significantly lower averaged HIs in the PTV for SIB 


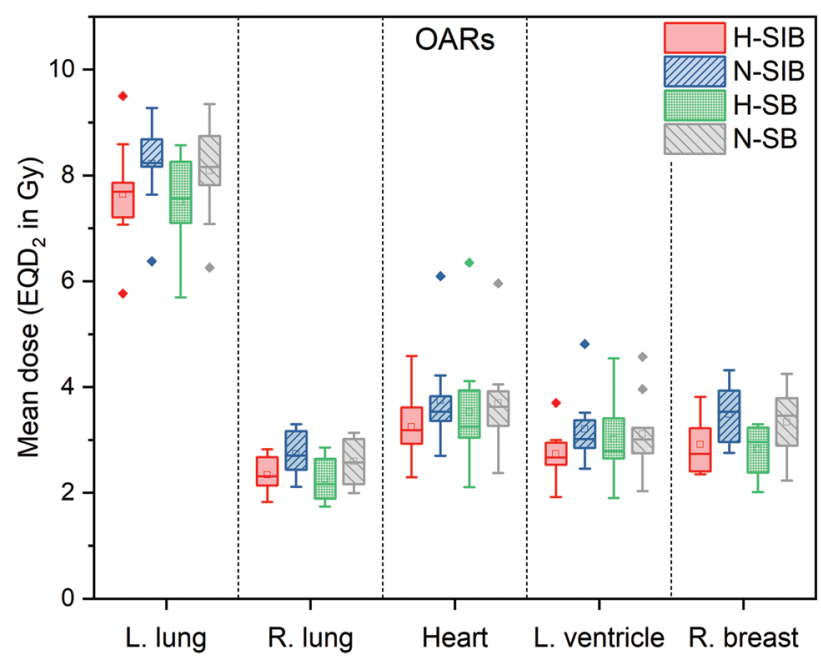

Figure 2. Box plot (the small square within the boxes indicates the average value, the line within the boxes indicates the median value, the box the range from 25 th to 75 th percentile, the whiskers the range from minimum to maximum and the rhombs the outliers) comparing the planned mean dose (EQD2) of the left $(L)$ and right $(R)$ lung, the heart, the left ventricle and the ipsilateral breast over all patients for all regarded treatment schemes for left-sided breast irradiation. $H$-SIB: Hypofractionated radiotherapy with simultaneous integrated boost (SIB); N-SIB: normofractionated radiotherapy with SIB; $H-S B$ : hypofractionated radiotherapy with sequential boost $(S B) ; N-S B$ : normofractionated radiotherapy with $S B$.

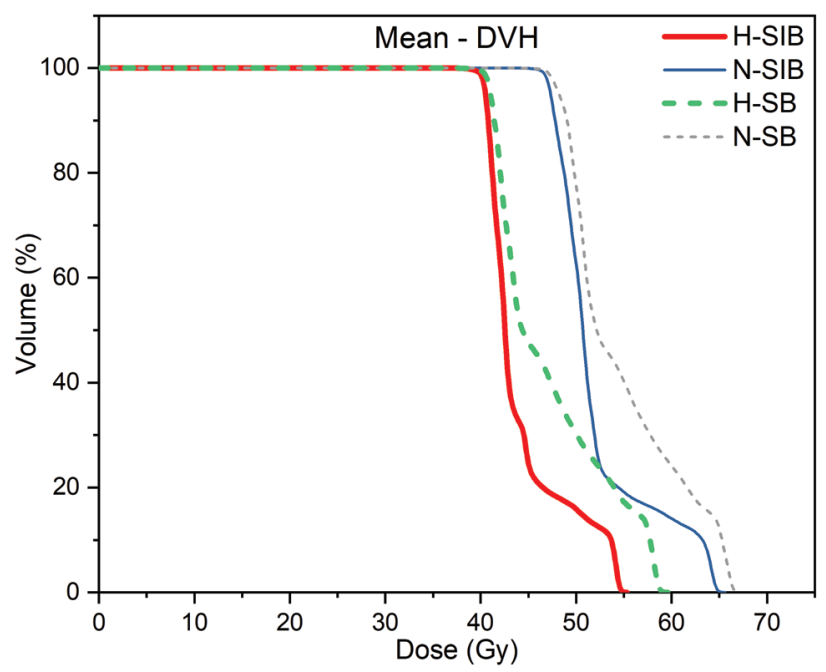

Figure 3. Comparison of the dose-volume histogram (DVH). Averaged physical doses for the planning target volume of the treated breast including the boost volume (PTVIB) of all patients for all examined treatment schemes. H-SIB: Hypofractionated radiotherapy with simultaneous integrated boost (SIB), N-SIB: normofractionated radiotherapy with $S I B, H-S B$ : hypofractionated radiotherapy with sequential boost (SB), $N$-SB: normofractionated radiotherapy with $S B$.

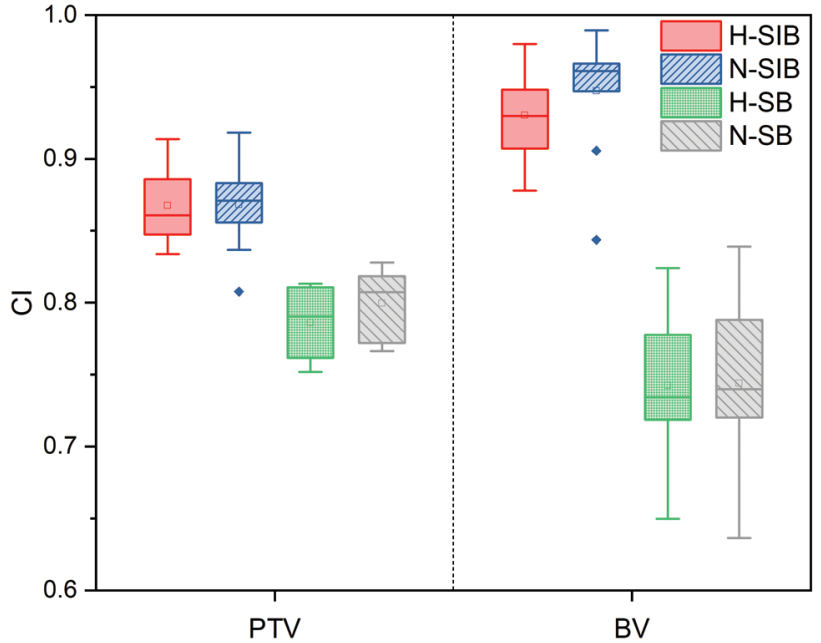

Figure 4. Box plot (the small square within the boxes indicates the average value, the line within the boxes indicates the median value, the box the range from $25^{\text {th }}$ to $75^{\text {th }}$ percentile, the whiskers the range from minimum to maximum and the rhombs the outliers) comparing the conformity index $(C I)$ of the planning target volume (PTV) of the treated breast (left part) and boost volume (BV) (right part) of all patients for all examined treatment schemes. H-SIB: Hypofractionated radiotherapy with simultaneous integrated boost (SIB), N-SIB: normofractionated radiotherapy with SIB, H-SB: hypofractionated radiotherapy with sequential boost $(S B), N-S B$ : normofractionated radiotherapy with $S B$.

plans (N-SIB: $0.205 \pm 0.022$ (standard deviation); H-SIB: $0.225 \pm 0.008$ ) than for SB plans (N-SB: $0.288 \pm 0.010$; H-SB: $0.336 \pm 0.012 ; p<0.001)$. For the $\mathrm{BV}$, the $\mathrm{SB}$ technique resulted in significantly better homogeneity of dose distribution. Comparing the HIs of the PTV and BV with each other, distinctly lower averaged values were found for the $\mathrm{BV}$ in all plans (e.g. N-SB PTV: 0.288 \pm 0.01 vs. BV: 0.0251 \pm 0.0051 , $p<0.001)$. N-SIB and H-SIB showed slight but not significant, differences in homogeneity for both PTV and BV.

$P T V, B V$, and shell. The data presented in Figure 6 and Table III show the effect of different $\alpha / \beta$ values on the mean dose to the PTV. For the H-SIB and H-SB plans, the mean $\mathrm{EQD}_{2}$ dose increased with decreasing $\alpha / \beta$; the opposite pattern was observed in the N-SIB and N-SB plans. This correlation arises from the reference fraction dose of $2 \mathrm{~Gy}\left(\mathrm{EQD}_{2}\right)$. All regions with a fraction dose below this show an increase, while all regions with a fraction dose above it show a decrease when $\alpha / \beta$ increases. Depending on the difference between the original and reference fraction doses, this effect is more or less pronounced. Comparing the normofractionated plans with one another and hypofractionated plans with one 
ANTICANCER RESEARCH 41: 1909-1920 (2021)

Table II. Averaged conformity index and homogeneity index with standard deviation for both, the planning target volume of the treated breast and boost volume, of all patients and all examined treatment schemes and the p-value of the paired t-test comparison with the H-SIB scheme.

\begin{tabular}{|c|c|c|c|c|c|c|c|c|}
\hline & \multicolumn{4}{|c|}{$\mathrm{CI}$} & \multicolumn{4}{|c|}{$\mathrm{HI}$} \\
\hline & PTV & $p$-Value & BV & $p$-Value & PTV & $p$-Value & $\mathrm{BV}$ & $p$-Value \\
\hline H-SIB & $0.868 \pm 0.027$ & --- & $0.930 \pm 0.032$ & --- & $0.225 \pm 0.008$ & --- & $0.0428 \pm 0.0042$ & --- \\
\hline N-SIB & $0.868 \pm 0.030$ & 0.895 & $0.947 \pm 0.043$ & 0.084 & $0.205 \pm 0.022$ & 0.049 & $0.0498 \pm 0.0077$ & 0.026 \\
\hline $\mathrm{H}-\mathrm{SB}$ & $0.786 \pm 0.024$ & $<0.001$ & $0.742 \pm 0.049$ & $<0.001$ & $0.336 \pm 0.012$ & $<0.001$ & $0.0246 \pm 0.0047$ & $<0.001$ \\
\hline $\mathrm{N}-\mathrm{SB}$ & $0.800 \pm 0.023$ & $<0.001$ & $0.744 \pm 0.055$ & $<0.001$ & $0.288 \pm 0.010$ & $<0.001$ & $0.0251 \pm 0.0051$ & $<0.001$ \\
\hline
\end{tabular}

H-SIB: Hypofractionated-simultaneous-integrated boost; N-SIB: normofractionated-simultaneous-integrated boost; H-SB: hypofractionated radiotherapy with sequential boost (SB); N-SB: normofractionated radiotherapy with SB; PTV: planning-target volume; BV: boost volume; CI: conformity index; HI: homogeneity index.

Table III. Averaged mean dose $\left(E Q D_{2}\right.$, calculated with different $\alpha / \beta$ values) with standard deviation of the planning target volume, boost volume, and shell of all patients and all examined treatment schemes and the p-value of the paired t-test comparison with the H-SIB scheme.

\begin{tabular}{|c|c|c|c|c|c|c|c|c|}
\hline & \multicolumn{8}{|c|}{ PTV mean dose (Gy) } \\
\hline & {$[\alpha / \beta]=2.2$} & $p$-Value & {$[\alpha / \beta]=3$} & $p$-Value & {$[\alpha / \beta]=3.8$} & $p$-Value & {$[\alpha / \beta]=4.6$} & $p$-Value \\
\hline H-SIB & $49.96 \pm 0.39$ & --- & $48,84 \pm 0.36$ & --- & $48.03 \pm 0.34$ & --- & $47.42 \pm 0.33$ & --- \\
\hline N-SIB & $48.47 \pm 0.34$ & $<0.001$ & $48,83 \pm 0.32$ & 0.910 & $49.10 \pm 0.31$ & $<0.001$ & $49.29 \pm 0.30$ & $<0.001$ \\
\hline $\mathrm{H}-\mathrm{SB}$ & $50.88 \pm 0.54$ & $<0.001$ & $50.06 \pm 0.58$ & $<0.001$ & $49.47 \pm 0.61$ & $<0.001$ & $49.02 \pm 0.63$ & $<0.001$ \\
\hline \multirow[t]{3}{*}{ N-SB } & $49.57 \pm 0.63$ & 0.028 & $50.19 \pm 0.67$ & $<0.001$ & $50.64 \pm 0.69$ & $<0.001$ & $50.98 \pm 0.71$ & $<0.001$ \\
\hline & \multicolumn{8}{|c|}{ BV mean dose (Gy) } \\
\hline & {$[\alpha / \beta]=2.2$} & $p$-Value & {$[\alpha / \beta]=3$} & $p$-Value & {$[\alpha / \beta]=3.8$} & $p$-Value & {$[\alpha / \beta]=4.6$} & $p$-Value \\
\hline H-SIB & $71.85 \pm 0.25$ & --- & $69,00 \pm 0.23$ & --- & $66.94 \pm 0.21$ & --- & $65.38 \pm 0.20$ & --- \\
\hline N-SIB & $68,19 \pm 0.21$ & $<0.001$ & $67.50 \pm 0.20$ & $<0.001$ & $67.00 \pm 0.19$ & 0.384 & $66.63 \pm 0.18$ & $<0.001$ \\
\hline $\mathrm{H}-\mathrm{SB}$ & $64,54 \pm 0.29$ & $<0.001$ & $63.52 \pm 0.27$ & $<0.001$ & $62.78 \pm 0.26$ & $<0.001$ & $62.22 \pm 0.25$ & $<0.001$ \\
\hline \multirow[t]{3}{*}{$\mathrm{N}-\mathrm{SB}$} & $63.13 \pm 0.51$ & $<0.001$ & $63.56 \pm 0.48$ & $<0.001$ & $63.86 \pm 0.47$ & $<0.001$ & $64.09 \pm 0.46$ & $<0.001$ \\
\hline & \multicolumn{8}{|c|}{ Shell mean dose (Gy) } \\
\hline & {$[\alpha / \beta]=2.2$} & $p$-Value & {$[\alpha / \beta]=3$} & $p$-Value & {$[\alpha / \beta]=3.8$} & $p$-Value & {$[\alpha / \beta]=4.6$} & $p$-Value \\
\hline H-SIB & $58.30 \pm 0.69$ & --- & $56.56 \pm 0.64$ & --- & $55.30 \pm 0.60$ & --- & $54.35 \pm 0.57$ & --- \\
\hline N-SIB & $54.83 \pm 1.73$ & $<0.001$ & $54.89 \pm 1.64$ & 0.014 & $54.93 \pm 1.58$ & 0.500 & $54.97 \pm 1.53$ & 0.252 \\
\hline $\mathrm{H}-\mathrm{SB}$ & $57.42 \pm 0.92$ & 0.014 & $56.67 \pm 0.90$ & 0.705 & $56.13 \pm 0.88$ & 0.014 & $55.72 \pm 0.87$ & $<0.001$ \\
\hline N-SB & $56.03 \pm 1.11$ & $<0.001$ & $56.72 \pm 1.08$ & 0.634 & $57.22 \pm 1.05$ & $<0.001$ & $57.60 \pm 1.04$ & $<0.001$ \\
\hline
\end{tabular}

H-SIB: Hypofractionated-simultaneous-integrated boost; N-SIB: normofractionated-simultaneous-integrated boost; H-SB: hypofractionated radiotherapy with sequential boost (SB); N-SB: normofractionated radiotherapy with SB; PTV: planning-target volume; BV: boost volume.

another, the SIB plans generally resulted in a significantly lower averaged mean dose. The dependence on $\alpha / \beta$ was balanced for $\alpha / \beta=3$ Gy; no significant differences were obtained using hypofractionated versus normofractionated plans [e.g. N-SIB: $48.83 \pm 0.32$ Gy (standard deviation); HSIB: $48.84 \pm 0.36$ Gy, $p>0.05]$.

Evaluation of the BV (Figure 7 and Table III) clearly shows higher mean doses $\left(\mathrm{EQD}_{2}\right)$ for SIB plans across all $\alpha / \beta$ values. Notably, at $\alpha / \beta=2.2 \mathrm{~Gy}$, there was a difference of $11.3 \%$ (H-SIB $v s . \mathrm{H}-\mathrm{SB})$ and $8 \%(\mathrm{~N}-\mathrm{SIB} v s . \mathrm{N}-\mathrm{SB})$ in the mean dose. A direct comparison of all schemes against the H-SIB scheme shows a significantly lower averaged mean dose for both SB schemes (e.g. H-SIB: 66.94 $\pm 0.21 \mathrm{~Gy} v s$. H-SB: $62.78 \pm 0.26 \mathrm{~Gy}, \alpha / \beta=3.8 \mathrm{~Gy}, p<0.001)$. Compared with that for $\mathrm{H}-\mathrm{SIB}$, the N-SIB scheme also produced significantly lower results for $\alpha / \beta=2.2$ Gy and $\alpha / \beta=3$ Gy but showed similar results for $\alpha / \beta=3.8$ Gy and significantly higher results for $\alpha / \beta=4.6$ Gy (H-SIB: $65.38 \pm 0.2$ Gy $v s$. N- 


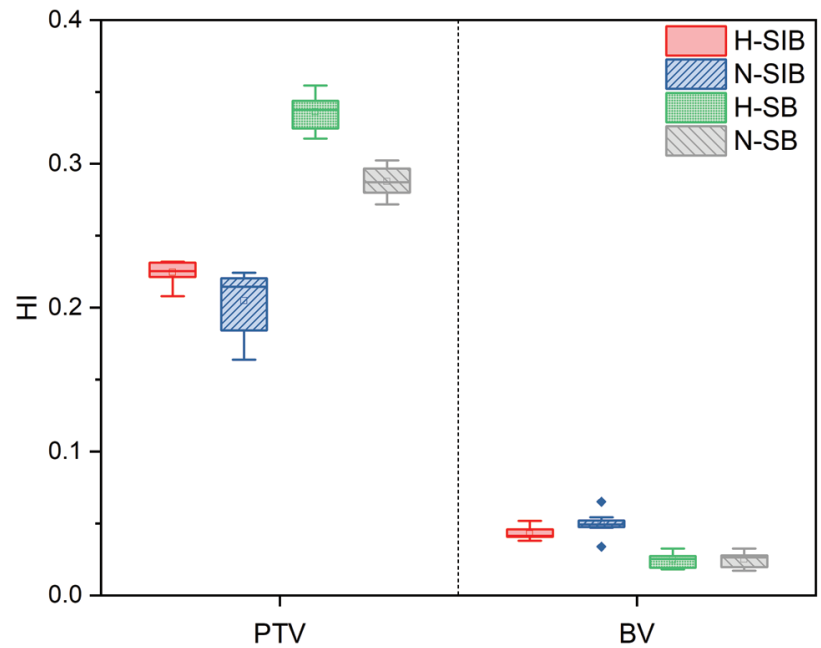

Figure 5. Box plot (the small square within the boxes indicates the average value, the line within the boxes indicates the median value, the box the range from $25^{\text {th }}$ to $75^{\text {th }}$ percentile, the whiskers the range from minimum to maximum and the rhombs the outliers) comparing the homogeneity index (HI) of the planning target volume (PTV) of the treated breast (left part) and boost volume (BV) (right part) of all patients for all examined treatment schemes. H-SIB: Hypofractionated radiotherapy with simultaneous integrated boost (SIB), N-SIB normofractionated radiotherapy with SIB, $H$-SB: hypofractionated radiotherapy with sequential boost $(S B), N$-SB: normofractionated radiotherapy with $S B$.

SIB: $66.63 \pm 0.18 \mathrm{~Gy}, p<0.001)$.

Figure 8 and Table III show the analysed data for the shell (1-cm-thick auxiliary structure around the BV to evaluate dose gradients). For $\alpha / \beta=2.2 \mathrm{~Gy}$, the H-SIB scheme had significantly higher averaged mean $\mathrm{EQD}_{2}$ values $(58.3 \pm 0.69$ Gy) than all other schemes (all $p<0.05$ ). The N-SIB plans resulted in the lowest mean dose and were still significantly lower for $\alpha / \beta=3$ Gy, while the H-SIB and both SB schemes produced comparable values. Higher $\alpha / \beta$ values led to significantly lower averaged mean doses in the H-SIB plans than in the other schemes, with a drop to a significantly lower point than in either SB scheme (e.g. H-SIB: $54.35 \pm 0.57$ Gy $v s$. H-SB: $55.60 \pm 1.04 \mathrm{~Gy}, \alpha / \beta=4.6 \mathrm{~Gy}$, $p<0.001)$. For $\alpha / \beta=3.8$ Gy and $4.6 \mathrm{~Gy}$, the N-SIB plans were similar to the H-SIB plans and no significant difference in averaged mean dose was observed (e.g. N-SIB: $54.93 \pm 1.58$ Gy $v s$. H-SIB: $55.3 \pm 0.6 \mathrm{~Gy}, \alpha / \beta=3.8 \mathrm{~Gy})$.

\section{Discussion}

This is a comparative plan study of different radiation techniques using helical tomotherapy with TomoEdge technology (H-SIB, N-SIB, H-SB, and N-SB) used to irradiate

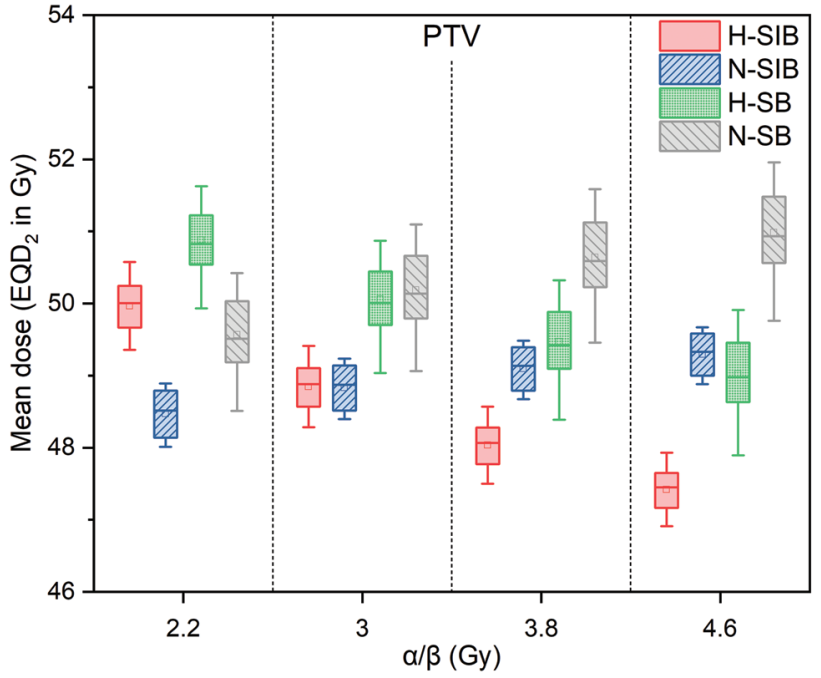

Figure 6. Box plot (the small square within the boxes indicates the average value, the line within the boxes indicates the median value, the box the range from 25th to 75 th percentile, the whiskers the range from minimum to maximum and the rhombs the outliers) comparing the mean dose $\left(E Q D_{2}\right)$ of the planning target volume (PTV) of the treated breast of all patients for all examined treatment schemes assuming four different values of $\alpha / \beta$. The $\alpha / \beta$ values are arranged and start with the lowest assumed value on the left and end with the highest on the right. H-SIB: Hypofractionated radiotherapy with simultaneous integrated boost (SIB), N-SIB: normofractionated radiotherapy with SIB, $H$-SB: hypofractionated radiotherapy with sequential boost (SB), N-SB: normofractionated radiotherapy with $S B$.

the left breast and include an indication for a radiation boost following breast-conserving surgery for breast cancer. Ten patients were included, and the results of interest are outlined below. First, we were able to show that radiation exposure of surrounding OAR, such as the contralateral breast, heart, and lungs, can be significantly reduced by implementing a hypofractionated radiation method at mid-EQD ${ }_{2}$. No significant difference was observed between the two hypofractionated groups (H-SIB and H-SB).

Further, the data produced by this study show that using a simultaneous integrated boost, whether normo- or hypofractionated, always produces greater conformity in both the PTV and the boost volume (BV). No significant difference was observed between the two SIB groups (H-SIB and N-SIB) in this regard. The improvement in conformity achieved by using a simultaneous integrated boost also induces a steep dose gradient within the PTV moving to the BV. This greatly reduces the excess dose burden to the PTV. On the other hand, both hypofractionated techniques produced more inhomogeneous dosing within both the PTV and BV.

In the literature, analyses of mean equivalent dose $\left(\mathrm{EQD}_{2}\right)$ to breast cancer tissue have not produced a consensus regarding the precise $\alpha / \beta$ value, with acceptable options 


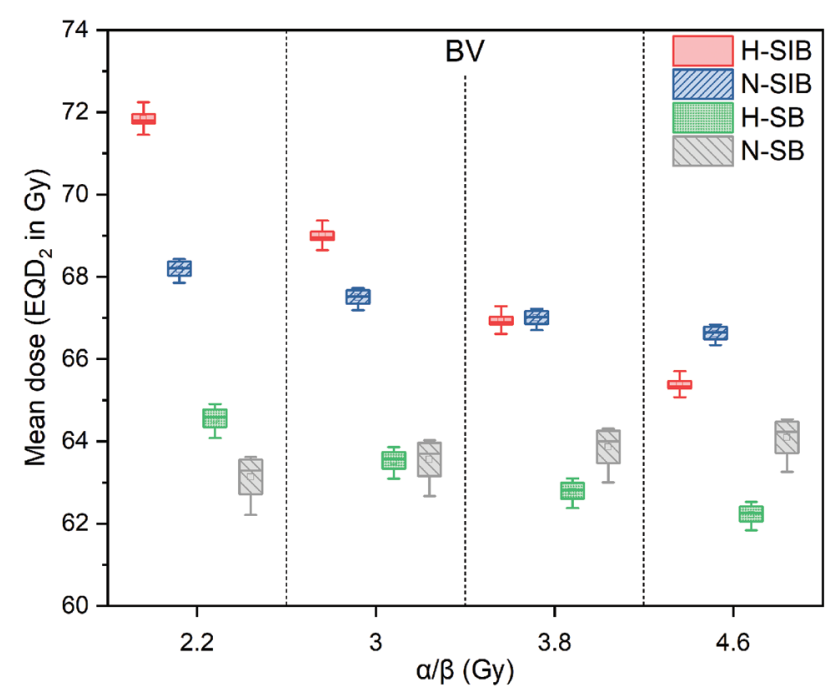

Figure 7. Box plot (the small square within the boxes indicates the average value, the line within the boxes indicates the median value, the box the range from $25^{\text {th }}$ to $75^{\text {th }}$ percentile, the whiskers the range from minimum to maximum and the rhombs the outliers) comparing the mean dose $\left(E Q D_{2}\right)$ of the boost volume $(B V)$ of all patients and all examined treatment schemes assuming four different values of $\alpha / \beta$. The $\alpha / \beta$ values are arranged and start with the lowest assumed value on the left and end with the highest on the right. H-SIB: Hypofractionated radiotherapy with simultaneous integrated boost (SIB), N-SIB: normofractionated radiotherapy with $S I B, H-S B$ : hypofractionated radiotherapy with sequential boost ( $S B$ ), $N$-SB: normofractionated radiotherapy with $S B$.

ranging from 2.2 to $4.6 \mathrm{~Gy}$ (20). One main explanation for the reported heterogeneity of reported $\alpha / \beta$ values is the different histological types and subtypes of breast cancer tissue. For this reason, calculations were carried out using different $\alpha / \beta$ values across the accepted range $(2.2 \mathrm{~Gy}, 3 \mathrm{~Gy}$, $3.8 \mathrm{~Gy}$, and $4.6 \mathrm{~Gy})$. This applies to the analysis of the mean equivalent dose to the PTV, BV, and shell $(1-\mathrm{cm}$ border around the BV). For the PTV, dose application using simultaneous integrated boost, both normo- and hypofractionated, showed mean $\mathrm{EQD}_{2}$ close to $50 \mathrm{~Gy}$ and below for all assumed $\alpha / \beta$ values tested. In contrast, techniques using sequential boost application tended to result in a mean $\mathrm{EQD}_{2}$ above $50 \mathrm{~Gy}$. For the H-SIB and $\mathrm{H}-\mathrm{SB}$ plans, the mean $\mathrm{EQD}_{2}$ dose increased parallel to decreasing $\alpha / \beta$; the opposite behaviour was observed in the N-SIB and $\mathrm{N}$-SB plans. Overall, the $\mathrm{EQD}_{2}$ in the PTV in all cases dropped within a range in which adequate local control and no increase in clinical side effects would be expected $(2,3)$. In the $1 \mathrm{~cm}$-border around the boost volume (the shell), which falls within the PTV, we observed that sequential boost radiation produced almost identical mean $\mathrm{EQD}_{2}$ values, i.e. between 55 and $57.5 \mathrm{~Gy}$, for a range of assumed

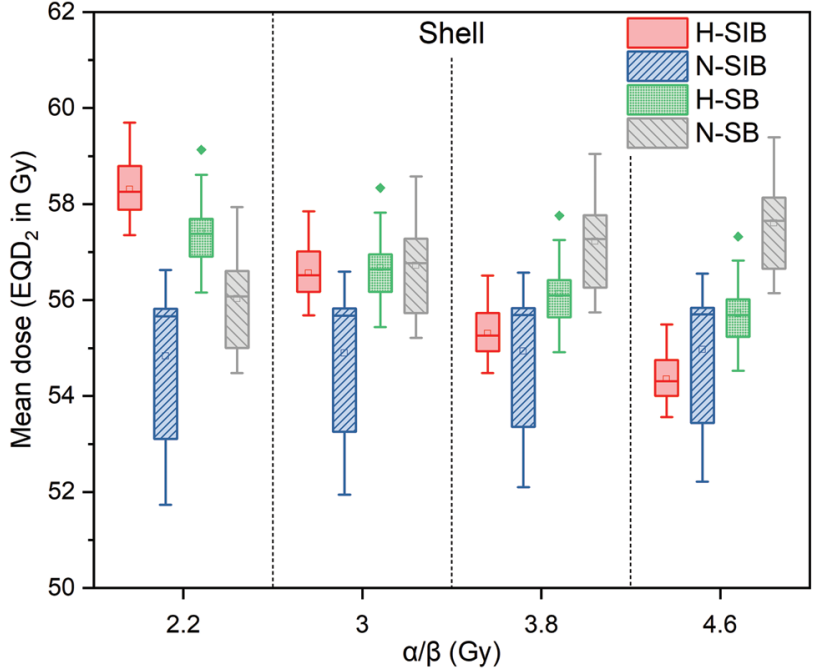

Figure 8. Box plot (the small square within the boxes indicates the average value, the line within the boxes indicates the median value, the box the range from $25^{\text {th }}$ to $7^{\text {th }}$ percentile, the whiskers the range from minimum to maximum and the rhombs the outliers) comparing the mean dose $\left(E Q D_{2}\right)$ of the shell (a 1-cm thick auxiliary structure around the boost volume) of all patients and all examined treatment schemes assuming four different values of $\alpha / \beta$. The $\alpha / \beta$ values are arranged and start with the lowest assumed value on the left and end with the highest on the right. H-SIB: Hypofractionated radiotherapy with simultaneous integrated boost (SIB), N-SIB: normofractionated radiotherapy with $S I B, H$-SB: hypofractionated radiotherapy with sequential boost (SB), $N-S B$ : normofractionated radiotherapy with $S B$.

$\alpha / \beta$ values, regardless of whether the radiation was normoor hypofractionated. The normofractionated SIB group consistently had low $\mathrm{EQD}_{2}$ values in the shell, lower than 56 Gy, across all assumed $\alpha / \beta$ values. The H-SIB method showed strong dependence on the mean $\mathrm{EQD}_{2}$ in the shell volume. At an $\alpha / \beta$ value of 2.2 , the median $\mathrm{EQD}_{2}$ was over $58 \mathrm{~Gy}$. This decreased continuously as the value of $\alpha / \beta$ increased. At an $\alpha / \beta$ value of $4.6 \mathrm{~Gy}$, the median $\mathrm{EQD}_{2}$ was only $54 \mathrm{~Gy}$. The optimum goal would be a median $\mathrm{EQD}_{2}$ as close as possible to $50 \mathrm{~Gy}$ in the PTV and shell volume (2, $3)$. This would provide sufficient cancer control whilst minimising the risks of fibrosis and fat necrosis in the shell. Even independently of the different assumed $\alpha / \beta$ values, all investigated radiation methods produced comparable exposure doses in the PTV and shell. Therefore, similar rates of tumour control and side effects would be expected here.

An analysis of the BV showed that both the normo- and hypofractionated SB groups achieved relatively stable median $\mathrm{EQD}_{2}$ values of 63-65 Gy regardless of the assumed $\alpha / \beta$ value. Compared to the SB groups, the SIB groups had significantly higher mean $\mathrm{EQD}_{2}$ values but a proportional inverse relationship to increasing assumed $\alpha / \beta$ values (from 
2.2 to $4.6 \mathrm{~Gy}$ ) (20). One may postulate that SIB, particularly $\mathrm{H}$-SIB, may achieve improved local control in the BV. However, the risk of late-onset side effects, such as fibrosis or fat necrosis, may also increase $(10,13)$. Confirmation of long-term results in clinical, prospective, randomised trials is required to evaluate these issues.

Among the different methods for intensity-modulated radiotherapy (IMRT), helical tomotherapy stands out for its highly conformal and homogeneous dose distribution $(24,25)$. Therefore, helical tomotherapy often results in better conformity and homogeneity index scores than other IMRT techniques, such as step-and-shoot IMRT and volumemodulated arc therapy (VMAT) IMRT $(33,34)$. These indices are important in protecting normal tissue and achieving optimum dose application in the tumour region $(11,31)$. Moving onto dose coverage in the PTV and BV, helical tomotherapy with the TomoEdge technique generally complies with the specification for every volume fraction to receive at least $95 \%$ and less than $107 \%$ of the prescribed dose (29). According to the ICRU guidelines, compliance with this specification is a prerequisite for achieving the primary goal of optimal cancer control while also minimising toxicity rates. These specifications could be met using all plans calculated in this study, regardless of the boost application type and fractionation. The results of this study show that applying a simultaneous integrated boost using helical IMRT, whether hypofractionated or normofractionated, achieved significantly higher CI values than sequential boost application techniques. This was the case for both the PTV and BV. Regarding the shell region within the PTV, i.e. the 1 -cm-thick volume surrounding the $\mathrm{BV}$, we showed that N-SIB always produced the lowest median $\mathrm{EQD}_{2}$ values in the range of assumed $\alpha / \beta$ values, although the $\mathrm{EQD}_{2}$ always exceeded $50 \mathrm{~Gy}$. At low $\alpha / \beta$ values, the H-SIB was not significantly worse than $\mathrm{H}-\mathrm{SB}$. With increasing $\alpha / \beta$ values, the H-SIB was improving, as $\mathrm{N}$ SIB did. Comparing H-SIB to the other boost techniques for mean $\mathrm{EQD}_{2}$ in the PTV (excluding the $\mathrm{BV}$ ), we found that this method produces doses of $50 \mathrm{~Gy}(\alpha / \beta=2.2 \mathrm{~Gy})$, with lower doses at increasing $\alpha / \beta$ values. As far as we are aware, there are no equivalent analyses of boost application procedures in adjuvant breast radiation using other IMRT techniques, such as standard IMRT or VMAT, alongside their dependence on different assumed $\alpha / \beta$ values.

Based on our results, we would not expect that the use of H-SIB using helical IMRT with the TomoEdge technique results in different tumour control rates or in an increase of late-onset toxicity risks in the PTV (excluding the BV) compared with that observed in other treatment schemes.

Regarding the BV, SIB generally led to significantly higher $\mathrm{EQD}_{2}$ for all tested $\alpha / \beta$ values. This was highest using H-SIB at an assumed $\alpha / \beta$ value of 2.2 Gy and reduced as $\alpha / \beta$ values increased. Clinically, this could mean that improved long-term local control in the BV would be more likely when using H-SIB and N-SIB than employing sequential boost techniques. However, increased rates of late-onset toxicities, such as fibrosis or fat necrosis, are also possible. No long-term results are available for the phase I and II studies published thus far.

Currently, clinical data are available from individual phase I and II studies investigating the acute toxicity of hypofractionated adjuvant breast radiotherapy using simultaneous integrated boost (H-SIB) following breastconserving surgery. These studies used conventional tangential beam 3D techniques (3D-CRT), step-and-shoot IMRT techniques, and VMAT and reported only mild early onset toxicities (35-38). Long-term results of randomised prospective phase III studies are needed before a clinical statement can be made regarding whether H-SIB produces higher toxicity rates than the gold standard, N-SB or H-SB, or in comparison to N$\mathrm{SIB}$, which is also well established in routine clinical use.

A study by van Parjis et al. investigated a total of 70 patients and compared side effect rates from N-SB using 3DCRT to that from H-SIB using helical IMRT (tomotherapy) (39). The shortest follow-up period was 1 year and the longest was 3 years. H-SIB use showed a significant reduction in skin and lung toxicity after 2 years when compared to N-SB. The left ventricular ejection fraction was not significantly different. One limitation of this study is that the conventional 3D radiation method was compared with the highly conformal IMRT method, which facilitates very steep dose gradients. This means that the better results could be explained simply by the difference in quality between the two radiation methods rather than the type of fractionation. It should also be noted that the helical IMRT method used at the time was not the TomoEdge method, which was only developed later. The TomoEdge technique reduces the longitudinal dose exposure in normal tissue and can significantly reduce the treatment duration (29).

Paelinck et al. published a prospective randomised study comparing the early onset toxicities of H-SIB and H-SB using the same IMRT technique (VMAT). No significant difference was found in the primary endpoint of moist epitheliolysis. H-SIB produced significantly better results regarding the secondary endpoints of dermatitis and itching (40). For this study, patients were irradiated in the nonstandard prone position, which makes the data difficult to compare with other datasets.

Currently, clinically sufficient data from randomised, prospective, phase III studies on late-onset toxicity comparing $\mathrm{H}-\mathrm{SIB}$ to $\mathrm{N}-\mathrm{SB}, \mathrm{H}-\mathrm{SB}$, or $\mathrm{N}-\mathrm{SIB}$ are not available. The aim should be to use a period of 5-10 years to draw conclusions that could change therapy standards.

Only two investigations on this topic are available, and both have limited informative value. De Rose et al. carried out a retrospective analysis of $\mathrm{H}-\mathrm{SIB}$ in 831 patients, describing a relatively high rate of skin reactions after 2 years; however, 
these results were not confirmed after 5 years. The IMRT technique used was VMAT (41). Another study compared NSIB to H-SIB using tangential beam 3D-CRT in both cases. After an average follow-up period of 7.2 years, patients were asked about their satisfaction with the cosmetic results. This study found a positive tendency toward H-SIB. No objective data on toxicity rates were collected (42).

$\mathrm{H}-\mathrm{SIB}$ is currently an experimental technique in adjuvant radiotherapy for breast cancer and is under review in a large phase III study (HYPOSIB; NCT02474641). Valid clinical data on late-onset toxicities are therefore awaited. The data from our plan study do not show any obvious indications that would lead us to expect an increase in late-onset toxicities after H-SIB compared to currently used boost application methods. This statement is valid when radiation is applied using helical tomotherapy with TomoEdge technology. We can assume that this may also apply in principle to other IMRT techniques, although this should be confirmed by appropriate studies.

Further investigations are also needed to reduce the uncertainty regarding $\alpha / \beta$ values of breast cancer tissue in the literature and thus improve estimates of the radiation effect.

\section{Conclusion}

This study was the first to use a plan comparison to contrast hypofractionated radiotherapy with simultaneousintegrated boost, currently an experimental technique, with other common non-invasive methods for boost application (H-SB, N-SB, and N-SIB) in adjuvant radiotherapy using cutting-edge helical tomotherapy with TomoEdge technology following breast-conserving surgery. The dosimetric results provided no evidence that H-SIB would lead to different results regarding tumour control or lateonset side effects compared to other clinically standard treatment schemes when using helical tomotherapy with TomoEdge technology. Uncertainty still exists regarding the $\alpha / \beta$ values of breast cancer tissue, which was taken into account for our analysis. Long-term clinical results are awaited from randomised trials.

\section{Conflicts of Interest}

The Authors declare that there are no conflicts of interest in relation to this study.

\section{Authors' Contributions}

F.Z., M.S. and S.H. initiated and supervised the project. F.Z. and M.S. collected the data. M.S., F.Z., and C.K. performed the data analysis. M.S., F.Z., P.H., J.D. and S.H. interpreted the experimental data and were responsible for creating figures. F.Z. and M.S. wrote the manuscript with input from all Authors. All Authors have been involved in manuscript revisions.

\section{References}

1 Ghoncheh M, Pournamdar Z and Salehiniya H: Incidence and mortality and epidemiology of breast cancer in the world. Asian Pac J Cancer Prev 17(S3): 43-46, 2016. PMID: 27165206. DOI: 10.7314/apjcp.2016.17.s3.43

2 Clarke M, Collins R, Darby S, Davies C, Elphinstone P, Evans V, Godwin J, Gray R, Hicks C, James S, MacKinnon E, McGale P, McHugh T, Peto R, Taylor C, Wang Y and Early Breast Cancer Trialists' Collaborative Group (EBCTCG).: Effects of radiotherapy and of differences in the extent of surgery for early breast cancer on local recurrence and 15-year survival: An overview of the randomised trials. Lancet 366(9503): 2087-2106, 2005. PMID: 16360786. DOI: 10.1016/S0140-6736(05)67887-7

3 Early Breast Cancer Trialists' Collaborative Group (EBCTCG)., Darby S, McGale P, Correa C, Taylor C, Arriagada R, Clarke M, Cutter D, Davies C, Ewertz M, Godwin J, Gray R, Pierce L, Whelan T, Wang Y and Peto R: Effect of radiotherapy after breastconserving surgery on 10-year recurrence and 15-year breast cancer death: Meta-analysis of individual patient data for 10,801 women in 17 randomised trials. Lancet 378(9804): 1707-1716, 2011. PMID: 22019144. DOI: 10.1016/S0140-6736(11)61629-2

4 Bartelink H, Maingon P, Poortmans P, Weltens C, Fourquet A, Jager J, Schinagl D, Oei B, Rodenhuis C, Horiot JC, Struikmans H, Van Limbergen E, Kirova Y, Elkhuizen P, Bongartz R, Miralbell R, Morgan D, Dubois JB, Remouchamps V, Mirimanoff RO, Collette S, Collette L and European Organisation for Research and Treatment of Cancer Radiation Oncology and Breast Cancer Groups.: Whole-breast irradiation with or without a boost for patients treated with breastconserving surgery for early breast cancer: 20 -year follow-up of a randomised phase 3 trial. Lancet Oncol 16(1): 47-56, 2015. PMID: 25500422. DOI: 10.1016/S1470-2045(14)71156-8

5 Vrieling C, van Werkhoven E, Maingon P, Poortmans P, Weltens C, Fourquet A, Schinagl D, Oei B, Rodenhuis CC, Horiot JC, Struikmans H, Van Limbergen E, Kirova Y, Elkhuizen P, Bongartz R, Miralbell R, Morgan DA, Dubois JB, Remouchamps V, Mirimanoff RO, Hart G, Collette S, Collette L, Bartelink H and European Organisation for Research and Treatment of Cancer, Radiation Oncology and Breast Cancer Groups.: Prognostic factors for local control in breast cancer after long-term follow-up in the EORTC boost vs no boost trial: A RANDOMIZED CLINICAL TRIAL. JAMA Oncol 3(1): 4248, 2017. PMID: 27607734. DOI: 10.1001/jamaoncol.2016.3031

6 Aly MM, Abo-Madyan Y, Jahnke L, Wenz F and Glatting G: Comparison of breast sequential and simultaneous integrated boost using the biologically effective dose volume histogram (BEDVH). Radiat Oncol 11: 16, 2016. PMID: 26830843. DOI: 10.1186/s13014-016-0590-1

7 McDonald MW, Godette KD, Whitaker DJ, Davis LW and Johnstone PA: Three-year outcomes of breast intensitymodulated radiation therapy with simultaneous integrated boost. Int J Radiat Oncol Biol Phys 77(2): 523-530, 2010. PMID: 19775834. DOI: 10.1016/j.ijrobp.2009.05.042

8 Wenz F, Welzel G, Blank E, Hermann B, Steil V, Sütterlin M and Kraus-Tiefenbacher U: Intraoperative radiotherapy as a boost during breast-conserving surgery using low-kilovoltage $\mathrm{X}$-rays: the first 5 years of experience with a novel approach. Int J Radiat Oncol Biol Phys 77(5): 1309-1314, 2010. PMID: 20100643. DOI: $10.1016 /$ j.ijrobp.2009.06.085 
9 Veronesi U, Orecchia R, Luini A, Galimberti V, Zurrida S, Intra M, Veronesi P, Arnone P, Leonardi MC, Ciocca M, Lazzari R, Caldarella P, Rotmensz N, Sangalli C, Sances D and Maisonneuve $\mathrm{P}$ : Intraoperative radiotherapy during breast conserving surgery: A study on 1,822 cases treated with electrons. Breast Cancer Res Treat 124(1): 141-151, 2010. PMID: 20711810. DOI: 10.1007/s10549-010-1115-5

10 Michalski A, Atyeo J, Cox J, Rinks M, Morgia M and Lamoury G: A dosimetric comparison of 3D-CRT, IMRT, and static tomotherapy with an SIB for large and small breast volumes. Med Dosim 39(2): 163-168, 2014. PMID: 24393498. DOI: 10.1016/j.meddos.2013.12.003

11 Onal C, Efe E, Guler OC and Yildirim BA: Dosimetric comparison of sequential versus simultaneous-integrated boost in early-stage breast cancer patients treated with breastconserving surgery. In Vivo 33(6): 2181-2189, 2019. PMID: 31662554. DOI: 10.21873 /invivo. 11720

12 Van Parijs H, Reynders T, Heuninckx K, Verellen D, Storme G and De Ridder M: Breast conserving treatment for breast cancer: Dosimetric comparison of sequential versus simultaneous integrated photon boost. Biomed Res Int 2014: 827475, 2014 PMID: 25162031. DOI: 10.1155/2014/827475

13 Immink JM, Putter H, Bartelink H, Cardoso JS, Cardoso MJ, van der Hulst-Vijgen MHV, Noordijk EM, Poortmans PM, Rodenhuis CC and Struikmans H: Long-term cosmetic changes after breast-conserving treatment of patients with stage I-II breast cancer and included in the EORTC 'boost versus no boost' trial. Ann Oncol 23(10): 2591-2598, 2012. PMID: 22499858. DOI: 10.1093/annonc/mds066

14 Whelan TJ, Pignol JP, Levine MN, Julian JA, MacKenzie R, Parpia S, Shelley W, Grimard L, Bowen J, Lukka H, Perera F, Fyles A, Schneider K, Gulavita S and Freeman C: Long-term results of hypofractionated radiation therapy for breast cancer. N Engl J Med 362(6): 513-520, 2010. PMID: 20147717. DOI: 10.1056/NEJMoa0906260

15 START Trialists' Group., Bentzen SM, Agrawal RK, Aird EG, Barrett JM, Barrett-Lee PJ, Bentzen SM, Bliss JM, Brown J, Dewar JA, Dobbs HJ, Haviland JS, Hoskin PJ, Hopwood P, Lawton PA, Magee BJ, Mills J, Morgan DA, Owen JR, Simmons S, Sumo G, Sydenham MA, Venables K and Yarnold JR: The UK Standardisation of Breast Radiotherapy (START) Trial B of radiotherapy hypofractionation for treatment of early breast cancer: A randomised trial. Lancet 371(9618): 1098-1107, 2008. PMID: 18355913. DOI: 10.1016/S0140-6736(08)60348-7

16 Haviland JS, Owen JR, Dewar JA, Agrawal RK, Barrett J, BarrettLee PJ, Dobbs HJ, Hopwood P, Lawton PA, Magee BJ, Mills J, Simmons S, Sydenham MA, Venables K, Bliss JM, Yarnold JR and START Trialists' Group.: The UK Standardisation of Breast Radiotherapy (START) trials of radiotherapy hypofractionation for treatment of early breast cancer: 10-year follow-up results of two randomised controlled trials. Lancet Oncol 14(11): 1086-1094, 2013. PMID: 24055415. DOI: 10.1016/S1470-2045(13)70386-3

17 Brunt AM, Wheatley D, Yarnold J, Somaiah N, Kelly S, Harnett A, Coles C, Goodman A, Bahl A, Churn M, Zotova R, Sydenham M, Griffin CL, Morden JP, Bliss JM and FASTForward Trial Management Group.: Acute skin toxicity associated with a 1-week schedule of whole breast radiotherapy compared with a standard 3-week regimen delivered in the UK FAST-Forward Trial. Radiother Oncol 120(1): 114-118, 2016. PMID: 27046390. DOI: 10.1016/j.radonc.2016.02.027
18 Monten C, Lievens Y, Olteanu LAM, Paelinck L, Speleers B, Deseyne P, Van Den Broecke R, De Neve W and Veldeman L: Highly accelerated irradiation in 5 fractions (HAI-5): feasibility in elderly women with early or locally advanced breast cancer. Int J Radiat Oncol Biol Phys 98(4): 922-930, 2017. PMID: 28366576. DOI: 10.1016/j.ijrobp.2017.01.229

19 Murray Brunt A, Haviland JS, Wheatley DA, Sydenham MA, Alhasso A, Bloomfield DJ, Chan C, Churn M, Cleator S, Coles CE, Goodman A, Harnett A, Hopwood P, Kirby AM, Kirwan CC, Morris C, Nabi Z, Sawyer E, Somaiah N, Stones L, Syndikus I, Bliss JM, Yarnold JR and FAST-Forward Trial Management Group.: Hypofractionated breast radiotherapy for 1 week versus 3 weeks (FAST-Forward): 5-year efficacy and late normal tissue effects results from a multicentre, non-inferiority, randomised, phase 3 trial. Lancet 395(10237): 1613-1626, 2020. PMID: 32580883. DOI: 10.1016/S0140-6736(20)30932-6

20 van Leeuwen CM, Oei AL, Crezee J, Bel A, Franken NAP, Stalpers LJA and Kok HP: The alfa and beta of tumours: A review of parameters of the linear-quadratic model, derived from clinical radiotherapy studies. Radiat Oncol 13(1): 96, 2018. PMID: 29769103. DOI: 10.1186/s13014-018-1040-Z

21 Werner EM, Eggert MC, Bohnet S and Rades D: Prevalence and characteristics of pneumonitis following irradiation of breast cancer. Anticancer Res 39(11): 6355-6358, 2019. PMID: 31704867. DOI: $10.21873 /$ anticanres. 13847

22 Ahn S, Sung K, Kim HJ, Choi YE, Lee YK, Kim JS, Lee SK and Roh JY: Reducing radiation dermatitis using a film-forming silicone gel during breast radiotherapy: A pilot randomizedcontrolled trial. In Vivo 34(1): 413-422, 2020. PMID: 31882508. DOI: 10.21873 /invivo.11790

23 Tuohinen SS, Skytta T, Huhtala H, Virtanen V, KellokumpuLehtinen PL and Raatikainen P: Left ventricular speckle tracking echocardiography changes among early-stage breast cancer patients three years after radiotherapy. Anticancer Res 39(8): 4227-4236, 2019. PMID: 31366510. DOI: 10.21873/anticanres. 13584

24 Zwicker F, Swartman B, Roeder F, Sterzing F, Hauswald H, Thieke C, Weber KJ, Huber PE, Schubert K, Debus J and Herfarth K: In vivo measurement of dose distribution in patients' lymphocytes: Helical tomotherapy versus step-and-shoot IMRT in prostate cancer. J Radiat Res 56(2): 239-247, 2015. PMID: 25361548. DOI: $10.1093 / \mathrm{jrr} / \mathrm{rru} 096$

25 Altinok P, Tekçe E, Karaköse F, Berk K, Kalafat Ü, Kiziltan HS, Akgün Z and Mayadagli A: Dosimetric comparison of modern radiotherapy techniques for gastric cancer after total gastrectomy. J Cancer Res Ther 16(Supplement): S133-S137, 2020. PMID: 33380667. DOI: 10.4103/jcrt.JCRT_548_18

26 Krause S, Beck S, Schramm O, Schubert K, Hauswald H, Zabeldu Bois A, Herfarth K, Debus J and Sterzing F: Tomotherapy radiosurgery for arteriovenous malformations-current possibilities and future options with helical tomotherapy dynamic jaws? Technol Cancer Res Treat 12(5): 421-428, 2013. PMID: 23547977. DOI: 10.7785/tcrt.2012.500335

27 Welsh JS, Patel RR, Ritter MA, Harari PM, Mackie TR and Mehta MP: Helical tomotherapy: An innovative technology and approach to radiation therapy. Technol Cancer Res Treat 1(4): 311-316, 2002. PMID: 12625791. DOI: 10.1177/15330346 0200100413

28 Shepard DM, Olivera GH, Reckwerdt PJ and Mackie TR: Iterative approaches to dose optimization in tomotherapy. Phys 
Med Biol 45(1): 69-90, 2000. PMID: 10661584. DOI: 10.1088/0031-9155/45/1/306

29 Katayama S, Haefner MF, Mohr A, Schubert K, Oetzel D, Debus $\mathrm{J}$ and Sterzing F: Accelerated tomotherapy delivery with TomoEdge technique. J Appl Clin Med Phys 16(2): 4964, 2015. PMID: 26103170. DOI: 10.1120/jacmp.v16i2.4964

30 Chen M, Chen Y, Chen Q and Lu W: Theoretical analysis of the thread effect in helical TomoTherapy. Med Phys 38(11): 59455960, 2011. PMID: 22047359. DOI: 10.1118/1.3644842

31 Paddick I: A simple scoring ratio to index the conformity of radiosurgical treatment plans. Technical note. J Neurosurg 93(Suppl 3): 219-222, 2000. PMID: 11143252. DOI: 10.3171/ jns.2000.93.supplement

32 Fowler JF: 21 years of biologically effective dose. Br J Radiol 83(991): 554-568, 2010. PMID: 20603408. DOI: 10.1259/ bjr/31372149

33 Bortfeld T and Webb S: Single-Arc IMRT? Phys Med Biol 54(1): N9-20, 2009. PMID: 19075362. DOI: 10.1088/00319155/54/1/N02

34 Bartolucci L, Adrien C, Goudjil F, Amessis M, El Amine W, Fourquet A, Mazal A and Kirova Y: Dosimetric comparison of four high performance techniques for irradiation of breast cancer patients. Cancer Radiother : 2021. PMID: 33402289. DOI: 10.1016/j.canrad 2020.09 .001

35 Scorsetti M, Alongi F, Fogliata A, Pentimalli S, Navarria P, Lobefalo F, Garcia-Etienne C, Clivio A, Cozzi L, Mancosu P, Nicolini G, Vanetti E, Eboli M, Rossetti C, Rubino A, Sagona A, Arcangeli S, Gatzemeier W, Masci G, Torrisi R, Testori A, Alloisio M, Santoro A and Tinterri C: Phase I-II study of hypofractionated simultaneous integrated boost using volumetric modulated arc therapy for adjuvant radiation therapy in breast cancer patients: A report of feasibility and early toxicity results in the first 50 treatments. Radiat Oncol 7: 145, 2012. PMID: 22929062. DOI: 10.1186/1748-717X-7-145

36 Franco P, Zeverino M, Migliaccio F, Cante D, Sciacero P, Casanova Borca V, Torielli P, Arrichiello C, Girelli G, La Porta MR, Tofani S, Numico G and Ricardi U: Intensity-modulated and hypofractionated simultaneous integrated boost adjuvant breast radiation employing statics ports of tomotherapy (TomoDirect): A prospective phase II trial. J Cancer Res Clin Oncol 140(1): 167-177, 2014. PMID: 24292425. DOI: 10.1007/s00432-013-1560-8

37 Mondal D, Julka PK, Sharma DN, Jana M, Laviraj MA, Deo SV, Roy S, Guleria R and Rath GK: Accelerated hypofractionated adjuvant whole breast radiation with simultaneous integrated boost using volumetric modulated arc therapy for early breast cancer: A phase I/II dosimetric and clinical feasibility study from a tertiary cancer care centre of India. J Egypt Natl Canc Inst 29(1): 39-45, 2017. PMID: 28343538. DOI: 10.1016/j.jnci.2017.01.005
38 Krug D, Baumann R, Krockenberger K, Vonthein R, Schreiber A, Boicev A, Würschmidt F, Weinstrauch E, Eilf K, Andreas P, Höller U, Dinges S, Piefel K, Zimmer J, Dellas K and Dunst J: Adjuvant hypofractionated radiotherapy with simultaneous integrated boost after breast-conserving surgery: Results of a prospective trial. Strahlenther Onkol 197(1): 48-55, 2021. PMID: 33001241. DOI: 10.1007/s00066-020-01689-7

39 Van Parijs H, Miedema G, Vinh-Hung V, Verbanck S, Adriaenssens N, Kerkhove D, Reynders T, Schuermans D, Leysen K, Hanon S, Van Camp G, Vincken W, Storme G, Verellen D and De Ridder M: Short course radiotherapy with simultaneous integrated boost for stage I-II breast cancer, early toxicities of a randomized clinical trial. Radiat Oncol 7: 80, 2012. PMID: 22656865. DOI: 10.1186/1748-717X-7-80

40 Paelinck L, Gulyban A, Lakosi F, Vercauteren T, De Gersem W, Speleers B, Monten C, Mulliez T, Berkovic P, van Greveling A, Decoster F, Coucke P, De Neve W and Veldeman L: Does an integrated boost increase acute toxicity in prone hypofractionated breast irradiation? A randomized controlled trial. Radiother Oncol 122(1): 30-36, 2017. PMID: 28062087. DOI: $10.1016 /$ j.radonc.2016.12.023

41 De Rose F, Fogliata A, Franceschini D, Iftode C, D’Agostino GR, Comito T, Franzese C, Di Brina L, Clerici E, Loi M, Navarria P, Gatzemeier W, Testori A, Tinterri C, Lobefalo F, Tomatis S, Cozzi L and Scorsetti M: Hypofractionated whole breast irradiation and simultaneous integrated boost in largebreasted patients: Long-term toxicity and cosmesis. Clin Breast Cancer 20(6): 527-533, 2020. PMID: 32665192. DOI: 10.1016/j.clbc.2020.06.005

42 Lertbutsayanukul C, Pitak M, Ajchariyasongkram N, Rakkiet N, Seuree F and Prayongrat A: Long-term patient-rated cosmetic and satisfactory outcomes of early breast cancer treated with conventional versus hypofractionated breast irradiation with simultaneous integrated boost technique. Breast J 26(10): 19461952, 2020. PMID: 32648331. DOI: 10.1111/tbj.13960

Received February 15, 2021

Revised February 27, 2021 Accepted March 1, 2021 